\title{
A Petri net tool for software performance estimation based on upper throughput bounds
}

\author{
Ricardo J. Rodríguez ${ }^{1}$
}

Received: 12 July 2014 / Accepted: 17 November 2015 / Published online: 11 December 2015

(C) Springer Science+Business Media New York 2015

\begin{abstract}
Functional and non-functional properties analysis (i.e., dependability, security, or performance) ensures that requirements are fulfilled during the design phase of software systems. However, the Unified Modelling Language (UML), standard de facto in industry for software systems modelling, is unsuitable for any kind of analysis but can be tailored for specific analysis purposes through profiling. For instance, the MARTE profile enables to annotate performance data within UML models that can be later transformed to formal models (e.g., Petri nets or timed automatas) for performance evaluation. A performance (or throughput) estimation in such models normally relies on a whole exploration of the state space, which becomes unfeasible for large systems. To overcome this issue upper throughput bounds are computed, which provide an approximation to the real system throughput with a good complexityaccuracy trade-off. This paper introduces a tool, named Peabrain, that estimates the performance of software systems via their UML models. To do so, UML models are transformed to Petri nets where performance is estimated based on upper throughput bounds computation. Peabrain also allows to compute other features on Petri nets, such as the computation of upper and lower marking place bounds, and to simulate using an approximate (continuous) method. We show the applicability of Peabrain by evaluating the performance of a building closed circuit TV system.
\end{abstract}

Keywords Petri net $\cdot$ UML $\cdot$ Software performance $\cdot$ Optimization

Ricardo J. Rodríguez

rjrodriguez@unizar.es

1 Dpto. de Informática e Ingeniería de Sistemas, Universidad de Zaragoza, María de Luna 1, 50018 Saragossa, Spain 


\section{Introduction}

Software systems are normally modelled with Unified Modelling Language (UML) (OMG 2011b), the standard de facto in industry for software modelling. However, UML semantics is ambiguous (Fecher et al. 2005) and lacks for a support of formal validation (i.e., whether the system fulfils the requirements) and verification (i.e., whether the system fulfils internal correctness properties). Usually, verification and validation $(\mathrm{V} \& \mathrm{~V})$ are carried out in a system model focusing on the functional properties but avoiding non-functional properties. Examples of these non-functional properties are dependability, security or performance.

However, the system performance evaluation (or throughput, defined as completed jobs per unit of time), that is, to estimate how long the system takes to complete, is a primordial study in certain systems, mainly by its likely trade-off with other nonfunctional properties. For instance, let us suppose an embedded real-time distributed system of fire sensors deployed along a forest for early prevention of fire disasters. In this case, the system performance becomes crucial: the less the sensor activities take, the less battery consumption and thus a longer sensor useful life. This issue clearly evidences that the sooner the system performance is evaluated, the sooner it can be redesigned to get a better performance and save potential overruns. Note that the cost of redesigning a system in deployment has been quantified to be over the half of the total budget in certain domains (Randimbivololona 2001).

UML can be tailored for specific purposes through profiling (Selic 2007; Lagarde et al. 2007). A UML profile enriches UML models by extending its semantics. For instance, the Modelling and Analysis for Real-Time Embedded systems (MARTE) (OMG 2011a) profile provides an analysis framework called Quantitative Analysis Model (GQAM) that enables performance specification in UML models. A UML model with performance annotations (that is, UML enriched with MARTE profile) can be transformed to a formal model where performance evaluation is carried out, such as Stochastic Petri Nets (SPN) (Molloy 1982). Petri nets (PN) (Murata 1989), among other formal models, have been extensively studied in the literature as formal modelling language to evaluate UML models (the reader is referred to (Merseguer et al. 2002; López-Grao et al. 2004; Woodside et al. 2005; Koch and Parisi-Presicce 2006; Bernardi and Merseguer 2007; Berardinelli et al. 2009; Distefano et al. 2011), for naming a few).

However, a Petri net model of a software system can be so complex that exact performance evaluation becomes a highly complex computational task. The main reason for this complexity is the well-known state-space explosion problem. As a result, a task that requires an exhaustive state-space exploration becomes unachievable in a reasonable time for large systems. A way to overcome this problem is to estimate the performance by computing performance bounds (Campos and Silva 1992; Liu 1995; Rodríguez and Júlvez 2010; Rodríguez et al. 2013).

The computation of lower and upper throughput bounds gives an estimation about where the real system throughput is contained and can be a good approximation to future system performance improvement plans. This paper introduces an improvement of the tool Peabrain (Rodríguez et al. 2012), which implements previously published algorithms to compute lower and upper throughput bounds (Campos and 
Silva 1992; Rodríguez and Júlvez 2010; Rodríguez et al. 2013) in a Petri net. These algorithms intensively use linear programming (LP) techniques for which polynomial complexity algorithms exist, so they offer a good trade-off between accuracy and computational complexity.

Peabrain was initially presented in (Rodríguez et al. 2012), where a collection of modules for performance estimation and resource optimisation based on bounds computation for SPNs were introduced, as well as a SPN simulation analysis module based on exact Gillespie's stochastic simulation algorithm (Gillespie 1976). This paper introduces the plug-in architecture of Peabrain in more detail, and a complete description of its features. Besides, as case study we evaluate a building closed circuit TV system. The system is first modelled with UML, and annotated with MARTE to specify performance and resources data. Then, it is transformed to Petri net to carry a performance evaluation out using upper throughput bounds. We also show the benefit of applying this kind of computation instead of simulation techniques.

The plug-in architecture of Peabrain allows a modular extension of the tool, which helps to easily enhance the tool features. Peabrain was designed following structural and architectural patterns such as Facade and Model-View-Controller, respectively. Peabrain includes several enhancements with respect to (Rodríguez et al. 2012), such as the computation of lower and upper bounds for the average marking of places and the computation of structural implicit places. The Simulator module has been also improved in several ways: First, it computes the average marking of places; second, it creates an external file of results to make easier the handling after experiments; and third, a new simulation method, an approximate stochastic simulation algorithm (using Tau-Leaping method Gillespie 2007, 2008), has been implemented. Another interesting feature of Peabrain is the command-line execution, which enables the automation of evaluation by using scripts, and integration into batch executions.

Contribution In brief, the contribution of this paper is three-fold: First, we introduce a Petri net tool whose plug-in architecture enables a rapid prototype implementation of methods that use LP techniques on Petri nets. We exemplify it by extending Peabra iN with a module for computing structural implicit places; Second, an approximate simulation method faster than exact simulation is also introduced. Experiments show that this method performs better for nets with large number of resources; and third, we demonstrate how upper throughput bounds computation in a formal model, such as Petri nets, becomes useful to estimate the performance of a complex software system modelled with UML.

The outline of this paper is as follows. Section 2 reviews the literature about tools for performance evaluation in UML/PN models. Section 3 introduces some preliminary concepts needed to follow the rest of the paper, such as Petri nets, performance estimation based on bounds, and UML profiles. The plug-in architecture of PeabraiN and its last enhancements are explained in more detail in Sect. 4. Section 5 introduces the case study: A UML model annotated with MARTE of a building closed circuit TV system is transformed to a Petri net to estimate its performance using upper throughput bounds. Finally, Sect. 6 states some conclusions and future work. 


\section{Related work}

We have reviewed the literature focusing on tools that enable to evaluate performance in UML models or Petri nets and the tools that allow to compute upper/lower throughput bounds in Petri net models.

UML models can be transformed to different formal models, such as Petri nets or other timed automatas. In this work, we focus on tools that transform a UML model to Petri nets (Distefano et al. 2011). Some of these tools are ArgoPN (Delatour and de Lamotte 2003), ArgoPerformance (Distefano et al. 2011) or ArgoSPE (GómezMartínez and Merseguer 2006). The PN models obtained after transformation with these tools can be handled with different PN tools (such as GreatSPN Baarir et al. 2009 in the case of ArgoSPE) that compute the performance of the model through simulation, which becomes unfeasible for large systems due to the well-known state-space explosion problem. On the contrary, our tool enables not just simulation (exact and approximate methods) but also performance bound computation from which polynomial algorithms exist, thus offering a good complexity-accuracy trade-off.

To the best of our knowledge, the only tool for performance bound computation, is GreatSPN (Baarir et al. 2009), which computes lower and upper throughput bounds of transitions. GreatSPN is a tool for evaluation of performance in Petri nets widely used in the community. However, the programming language paradigm used for its implementation and its platform dependency makes very difficult to extend its functionalities, unlike Peabrain.

MATLAB (The MathWorks 2010) has also an optimisation package that can be used for LP computations. Nevertheless, it is a proprietary software and it depends of a proprietary software library (namely, MATLAB Component Runtime library) for a final software deployment. Besides, the representation of a PN in MATLAB is done in its mathematical form, what clearly makes the comprehension of the model at a glance harder. The extension of a MATLAB tool is neither not so straightforwardly.

Our tool, which is based on PIPE (Bonet et al. 2007) as we will show in the next section, has several benefits over the related tools: First, it allows an easy extension through modules; second, it uses the standard PN file format, Petri Net Markup Language (PNML) (Hillah et al. 2009), so it allows an interchange of files between different PNML-compliant tools; third, it facilitates a user-friendly GUI editor; and finally, it is a multi-platform and open source tool, which enables to be executed in different environments and its functionalities can be improved/revised by the community. Finally, it is worth also mentioning that PIPE enables to import standard performance format files, such as Performance Model Interchange Format (PMIF) (Smith et al. 2010). Therefore, a UML specification can be transformed to PMIF and then imported into PIPE (Lladó and Harrison 2011; Bonet and Lladó 2012), which clearly extends the usability of this tool.

\section{Preliminary concepts}

This section introduces concepts and references for the algorithms implemented in Peabrain, as well as it presents the UML profiles. 


\subsection{Petri nets}

A Petri net (Murata 1989) is a 4-tuple $\mathcal{N}=\langle P, T$, Pre, Post $\rangle$, where $P$ and $T$ are disjoint non-empty sets of places and transitions, and Pre (Post) are the pre-(post)incidence non-negative integer matrices of size $|P| \times|T|$. The pre-and post-set of a node $v \in P \cup T$ are respectively defined as $\bullet=\{u \in P \cup T \mid(u, v) \in F\}$ and $v^{\bullet}=\{u \in P \cup T \mid(v, u) \in F\}$, where $F \subseteq(P \times T) \cup(T \times P)$ is the set of directed arcs. A Petri net is said to be self-loop free if $\forall p \in P, t \in T t \in{ }^{\bullet} p$ implies $t \notin p^{\bullet}$. Ordinary nets are Petri nets whose arcs have weight 1. The incidence matrix of a Petri net is defined as $\mathbf{C}=$ Post - Pre.

A vector $\mathbf{m} \in \mathbb{Z}_{\geq 0}^{|P|}$ which assigns a non-negative integer to each place is called marking vector or marking. A Petri net system, or marked Petri net $\mathcal{S}=\left\langle\mathcal{N}, \mathbf{m}_{\mathbf{0}}\right\rangle$, is a Petri net $\mathcal{N}$ with an initial marking $\mathbf{m}_{\mathbf{0}}$.

A transition $t \in T$ is enabled at marking $\mathbf{m}$ if $\mathbf{m} \geq \operatorname{Pre}(\cdot, t)$, where $\operatorname{Pre}(\cdot, t)$ is the column of Pre corresponding to transition $t$. A transition $t$ enabled at $\mathbf{m}$ can fire yielding a new marking $\mathbf{m}^{\prime}=\mathbf{m}+\mathbf{C}(\cdot, t)$ (reached marking). This is denoted by $\mathbf{m} \stackrel{t}{\longrightarrow} \mathbf{m}^{\prime}$. A sequence of transitions $\sigma=\left\{t_{i}\right\}_{i=1}^{n}$ is a firing sequence in $\mathcal{S}$ if there exists a sequence of markings such that $\mathbf{m}_{\mathbf{0}} \stackrel{t_{1}}{\longrightarrow} \mathbf{m}_{1} \stackrel{t_{2}}{\longrightarrow} \mathbf{m}_{2} \ldots \stackrel{t_{n}}{\longrightarrow} \mathbf{m}_{n}$. In this case, marking $\mathbf{m}_{n}$ is said to be reachable from $\mathbf{m}_{\mathbf{0}}$ by firing $\sigma$, and this is denoted by $\mathbf{m}_{0} \stackrel{\sigma}{\longrightarrow} \mathbf{m}_{n}$. The enabling degree of a transition $t$ enabled at a marking $\mathbf{m}_{i}$, denoted as $\mathbf{e}_{\mathbf{m}_{i}}(t)$, is the biggest integer number $k$ such that $\mathbf{m}_{i} \geq k \cdot \operatorname{Pre}(\cdot, t)$. The firing count vector $\sigma \in \mathbb{Z}_{\geq 0}^{|T|}$ of the firable sequence $\sigma$ is a vector such that $\sigma(t)$ represents the number of occurrences of $t \in T$ in $\sigma$. If $\mathbf{m}_{\mathbf{0}} \stackrel{\sigma}{\longrightarrow} \mathbf{m}$, then we can write in vector form $\mathbf{m}=\mathbf{m}_{\mathbf{0}}+\mathbf{C} \cdot \boldsymbol{\sigma}$, which is referred to as the linear (or fundamental) state equation of the net.

Two transitions $t, t^{\prime}$ are said to be in structural conflict if they share, at least, one input place, i.e., ${ }^{\bullet} t \cap{ }^{\bullet} t^{\prime} \neq \emptyset$. Two transitions $t, t^{\prime}$ are said to be in effective conflict for a marking $\mathbf{m}$ if they are in structural conflict and they are both enabled at $\mathbf{m}$. Two transitions $t, t^{\prime}$ are in equal conflict if $\operatorname{Pre}(\cdot, t)=\operatorname{Pre}\left(\cdot, t^{\prime}\right) \neq \mathbf{0}$. The equal conflict relation is an equivalence relation that partitions the set of transitions into equivalence classes ECS, called equal conflict sets. Transitions belonging to a given equal conflict set are in extended free-choice conflict.

A Petri net is said to be strongly connected if there is a directed path joining any pair of nodes of the graph. A state machine is a particular type of ordinary Petri net where each transition has exactly one input arc and exactly one output arc, that is, $\left|t^{\bullet}\right|=\left.\right|^{\bullet} t \mid=1, \forall t \in T$.

A $p$-semiflow is a non-negative integer vector $\mathbf{y} \geq \mathbf{0}$ such that it is a left anuller of the net's incidence matrix, $\mathbf{y}^{\top} \cdot \mathbf{C}=0$. A p-semiflow implies a token conservation law independent from any firing of transitions. A $t$-semiflow is a non-negative integer vector $\mathbf{x} \geq \mathbf{0}$ such that is a right anuller of the net's incidence matrix, $\mathbf{C} \cdot \mathbf{x}=0$. A p- (or $\mathrm{t}$-)semiflow $\mathbf{v}$ is minimal when its support, $\|\mathbf{v}\|=\{i \mid \mathbf{v}(i) \neq 0\}$, is not a proper superset of the support of any other p- (or t-)semiflow, and the greatest common divisor of its elements is one. 
A place $p \in P$ is said to be implicit in $\mathcal{S}$ if it never is the unique place to constraint the occurrence of its output transitions. That is, an implicit place $p$ has enough tokens in any reachable marking of $\mathcal{S}$ such that the firing of its output transitions does not depends on marking of $p$. Thus, its removal does not affect the net system behaviour. A place $p$ is structurally implicit in $\mathcal{N}$ if for any initial marking $\mathbf{m}_{\mathbf{0}}$, the initial marking of $p$ can be chosen such that $p$ becomes implicit (i.e., $p$ can be removed from the net without affecting the the net behaviour). Algebraically, a place $p$ is structurally implicit if and only if there exits a p-semiflow $\mathbf{y}$ such that $\mathbf{y} \geq 0, \mathbf{y}(p)=0$ and $\mathbf{y}^{\top} \cdot \mathbf{C} \leq \mathbf{C}(p, \cdot)$. A structurally implicit place $p$ becomes implicit when its initial marking $\mathbf{m}_{\mathbf{0}}(p) \geq \max (0, z)$, where $z$ is solved by the following LPP (Garcia-Valles and Colom 1999):

$$
\begin{aligned}
& z=\text { minimize } \mathbf{y}^{\top} \cdot \mathbf{m}_{\mathbf{0}}+\mu \\
& \text { subject to } \mathbf{y}^{\top} \cdot \mathbf{C} \leq \mathbf{C}(p, \cdot) \\
& \mathbf{y}^{\top} \cdot \operatorname{Pre}(\cdot, t)+\mu \geq \operatorname{Pre}(p, t), \forall t \in p^{\bullet} \\
& \mathbf{y}(p)=0 \\
& \mathbf{y} \geq \mathbf{0}, \mu \geq 0
\end{aligned}
$$

Definition 1 (Silva and Colom 1988) Let be $\left\langle\mathcal{N}, \mathbf{m}_{\mathbf{0}}\right\rangle$ be a net system. The structural marking bound $N$ of a given place $p$ in $\mathcal{N}$ is

$$
\begin{aligned}
& N(p)=\text { maximum } \mathbf{m}(p) \\
& \text { subject to } \mathbf{m}=\mathbf{m}_{\mathbf{0}}+\mathbf{C} \cdot \boldsymbol{\sigma} \\
& \mathbf{m} \geq \mathbf{0}, \boldsymbol{\sigma} \geq \mathbf{0}
\end{aligned}
$$

A Stochastic Petri Net system (SPN) is a pair $\langle\mathcal{S}, \delta\rangle$ where $\mathcal{S}$ is a Petri net system and $\delta: T \rightarrow \mathbb{R}^{+}$is a positive real function such that $\delta(t)$ is the mean of the exponential firing time distribution associated to each transition $t \in T$. If $\delta(t)>0$, then transition $t$ is a timed transition. Otherwise, i.e., $\delta(t)=0$, transition $t$ is an immediate one. It will be assumed that all transitions in conflict are immediate.

The average marking vector, $\overline{\mathbf{m}}$, in an ergodic Petri net system is defined as (Florin and Natkin 1989):

$$
\overline{\mathbf{m}}(p) \underset{A S}{=} \lim _{\tau \rightarrow \infty} \frac{1}{\tau} \int_{0}^{\tau} \mathbf{m}(p)_{u} d u
$$

where $\mathbf{m}(p)_{u}$ is the marking of place $p$ at time $u$ and the notation $\overline{\overline{A S}}$ means equal almost surely.

Similarly, the steady-state throughput, $\chi$, in an ergodic Petri net is defined as (Florin and Natkin 1989):

$$
\chi(t)=\lim _{\tau \rightarrow \infty} \frac{\sigma(t)_{\tau}}{\tau}
$$

where $\sigma(t)_{\tau}$ is the firing count of transition $t$ at time $\tau$.

The vector of visit ratios expresses the relative throughput of transitions in the steady state. The visit ratio $\mathbf{v}(t)$ of each transition $t \in T$ normalised for transition $t_{i}$, 
$\mathbf{v}^{t_{i}}(t)$, is expressed as $\mathbf{v}^{t_{i}}(t)=\frac{\chi(t)}{\chi\left(t_{i}\right)}=\boldsymbol{\Gamma}\left(t_{i}\right) \cdot \chi(t), \forall t \in T$, where $\boldsymbol{\Gamma}\left(t_{i}\right)=\frac{1}{\chi\left(t_{i}\right)}$ represents the average inter-firing time of transition $t_{i}$ and $\chi(t)$ is the steady-state throughput of transition $t$.

In general, the vector of visit ratios $\mathbf{v}$ depends on the structure of the net, on the routing rates, on the initial marking $\mathbf{m}_{\mathbf{0}}$, and on the service times $\delta$ (Campos and Silva 1992). When the vector of visit ratios depends only on the structure of the net and on the routing rate, the Petri net is a Freely Related T-semiflows net (FRT-net) (Campos and Silva 1992). The range of FRT-nets is relatively broad. Examples of these kind of nets are mono-T-semiflow nets (i.e., nets having a single T-semiflow), choicefree nets, Process Petri nets (Tricas 2003), and nets where every conflict is an equal conflict. It is known that the continuous time Markov chain associated to these nets is ergodic (Campos and Silva 1992), what implies the existence of the above limits.

In a FRT-net, the vector of visit ratios $\mathbf{v}$ normalised for transition $t_{i}, \mathbf{v}^{t_{i}}$, can be calculated by solving the following linear system of equations (Campos and Silva 1992):

$$
\begin{aligned}
\left(\begin{array}{l}
\mathbf{C} \\
\mathbf{R}
\end{array}\right) \cdot \mathbf{v}^{t_{i}} & =0 \\
\mathbf{v}^{t_{i}}\left(t_{i}\right) & =1
\end{aligned}
$$

where $\mathbf{R}$ is a matrix containing the rates $\mathbf{r}(t)$ associated to transitions in equal conflict.

\subsection{Performance estimation based on bounds}

A lower bound for the average inter-firing time of transition $t_{i}, \Gamma^{\mathbf{l b}}\left(t_{i}\right)$, can be computed by solving the following LP problem (LPP) (Campos and Silva 1992):

$$
\begin{aligned}
& \boldsymbol{\Gamma}\left(t_{i}\right) \geq \boldsymbol{\Gamma}^{\mathbf{l b}}\left(t_{i}\right)=\operatorname{maximum} \mathbf{y}^{\boldsymbol{\top}} \cdot \operatorname{Pre} \cdot \mathbf{D}^{\mathbf{t}_{\mathbf{i}}} \\
& \text { subject to } \mathbf{y}^{\top} \cdot \mathbf{C}=\mathbf{0} \\
& \mathbf{y}^{\top} \cdot \mathbf{m}_{\mathbf{0}}=1 \\
& \mathbf{y} \geq \mathbf{0}
\end{aligned}
$$

where $\boldsymbol{\Gamma}\left(t_{i}\right)$ is the average interfiring time of transition $t_{i}$ and $\mathbf{D}^{\mathbf{t}_{\mathbf{i}}}$ is the vector of average service demands of transitions, $\mathbf{D}^{\mathbf{t}_{\mathbf{i}}}(t)=\mathbf{S}(t) \cdot \mathbf{v}^{t_{i}}(t)$ (the vector of visit ratios $\mathbf{v}^{t_{i}}$ is normalised for transition $t_{i}$ ). In the sequel, we omit the superindex $t_{i}$ in $\mathbf{D}^{\mathbf{t}_{\mathbf{i}}}$ for clarity.

As a side product of the solution of (6), $\mathbf{y}$ represents the slowest $\mathrm{p}$-semiflow of the system, thus LPP (6) can also be seen as a search for the most constraining p-semiflow. This p-semiflow will be the one with highest ratio $\frac{\mathbf{y}^{\top} \cdot \mathbf{P r e} \cdot \mathbf{D}}{\mathbf{y}^{\top} \cdot \mathbf{m}_{\mathbf{0}}}$. Therefore, an upper bound $\Theta\left(t_{i}\right)$ for the steady-state throughput can be calculated as the inverse of the lower bound for the average inter-firing time $\Gamma^{\mathbf{l b}}\left(t_{i}\right)$, that is, $\Theta\left(t_{i}\right)=\frac{1}{\Gamma^{\mathbf{l b}}\left(t_{i}\right)}$.

The LPP (6) can be applied when the vector of visit ratios $\mathbf{v}$ is known, as the computation of the vector of average service demands of transitions $\mathbf{D}$ is related to $\mathbf{v}$. 
After some manipulations, the LPP (6) can be transformed to its dual (Chiola et al. 1993), which can be used to compute an upper throughput bound $\Theta$ for a transition $t_{i}$ in a SPN:

$$
\begin{aligned}
& \chi\left(t_{i}\right) \leq \Theta\left(t_{i}\right)=\text { maximum } \chi\left(t_{i}\right) \\
& \text { subject to } \overline{\mathbf{m}}=\mathbf{m}_{\mathbf{0}}+\mathbf{C} \cdot \sigma \\
& \sum_{t \in{ }^{\bullet} p} \chi(t) \cdot \operatorname{Post}(p, t) \geq \sum_{t \in p^{\bullet}} \chi(t) \cdot \operatorname{Pre}(p, t), \forall p \in P \\
& \forall t \in T, \forall p \in{ }^{\bullet} t: \chi(t) \leq \frac{\overline{\mathbf{m}}(p)}{\delta(t) \cdot \operatorname{Pre}(p, t)} \\
& \forall t \in T,{ }^{\bullet} t=\{p\}: \chi(t) \cdot \delta(t) \geq \frac{\overline{\mathbf{m}}(p)-\operatorname{Pre}(p, t)+1}{\operatorname{Pre}(p, t)} \\
& \forall t \in T,{ }^{\bullet} t=\left\{p_{1}, p_{2}\right\}: \chi(t) \cdot \sigma(t) \cdot \operatorname{Pre}\left(p_{1}, t\right) \geq \\
& \overline{\mathbf{m}}\left(p_{1}\right)-\operatorname{Pre}\left(p_{1}, t\right)+ \\
& \quad 1-N\left(p_{1}\right) \cdot\left(1-\frac{\overline{\mathbf{m}}\left(p_{2}\right)-\operatorname{Pre}\left(p_{2}, t\right)+1}{N\left(p_{2}\right)-\operatorname{Pre}\left(p_{2}, t\right)+1}\right) \\
& \forall t_{j}, t_{k} \in E C S, r_{k} \cdot \chi\left(t_{j}\right)=r_{j} \cdot \chi\left(t_{k}\right) \\
& \mathbf{m}_{\mathbf{0}}, \sigma \geq \mathbf{0}, \chi(t) \geq 0, \forall t \in T
\end{aligned}
$$

Similarly, LPP (7) can be also used for computing the upper marking bound of a given place $p$ in $\mathcal{N}$, considering $\mathbf{m}(p)$ as objective function (instead of $\chi\left(t_{i}\right)$ ).

\subsection{UML profiles}

UML (OMG 2011b), the current standard modelling language for the industry and the software engineering research community, can be tailored for specific purposes through profiling (Selic 2007; Lagarde et al. 2007). A UML profile is a UML extension to enrich UML model semantics defined in terms of stereotypes (they are concepts in the target domain that will be added to the UML model), tags (the attributes of the stereotypes), and constraints (they are formulae that apply to stereotypes and UML elements to extend their semantics).

UML profiling has been a very active research field in the last years. As a matter of fact, several UML profiles can be found targeting at different domains and at analysis of non-functional properties (e.g., performance, dependability, or security). Examples of these UML profiles are Modelling and Analysis of Real-Time and Embedded systems (MARTE) (OMG 2011a), Dependability Analysis and Modelling (DAM) (Bernardi et al. 2011) and Security Analysis and Modelling (SecAM) (Rodríguez et al. 2010). These profiles enables to specify performance, dependability and security properties within UML models, respectively. In this paper, we use the MARTE profile to specify the activity timing in a UML model and to specify the number of resources in a system. Namely, we use the MARTE analysis frameworks called Generic Quantitative Analysis Model (GQAM) and the Generic Resource Modelling (GRM), which pro- 
vide stereotypes to these goals (gastep, hostDemand tagged value; resource, resMult tagged value, respectively).

\section{The Peabrain tool: architecture and features}

Peabrain, which stands for "Performance Estimation bAsed (on) Bounds (and) Resource optimisAtIon (for Petri) Nets", is made of a set of modules compliant with PIPE-tool modules (Bonet et al. 2007). In this section, we firstly introduce its architecture in detail and then the features provided by the tool.

\subsection{Architecture}

Peabra iN is programmed with Java and integrated on PIPE (Bonet et al. 2007). Aside from PIPE library dependencies, it depends on other libraries to perform its functionality. These libraries are related to computational operations in matrices (JAMA) (NIST 2012), probability distribution functions (SSJ) (Université de Montréal 2014), and LP solver-specific interface for Java (Java ILP) (JavaILP 2013).

Figure 1 depicts the UML Class diagram (UML-CD) of PeabraiN. It has been designed following the Model-View-Controller (MVC) architectural pattern, and its closed layered architecture is composed by three layers. Each layer corresponds with a

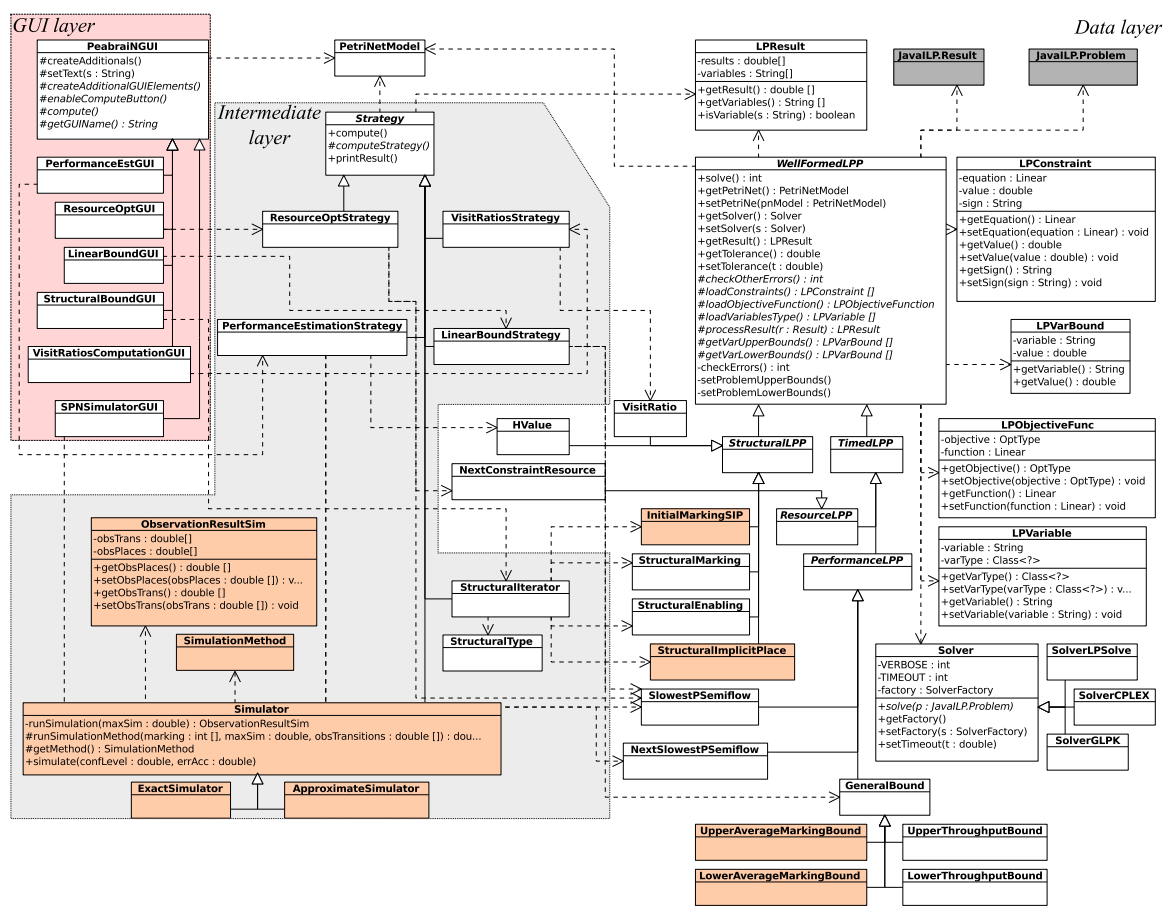

Fig. 1 UML Class diagram of PeabraiN 


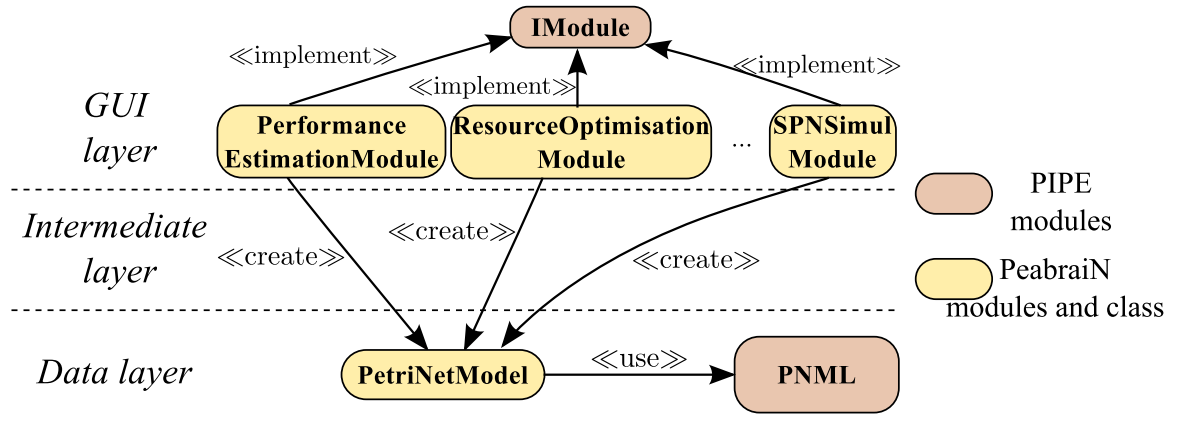

Fig. 2 Integration of Peabrain in the PIPE tool

component in MVC. The main classes added in the last release are depicted in orange colour. These classes are explained in more detail in Sect. 4.3.

The data layer contains classes representing the information needed for the provided functionalities to execute, in terms of LP problems (i.e., the constraints related to the LP problem, the definition of optimisation function, variable types, etc.). The Facade structural pattern has been followed in this layer to handle and minimise the classes complexity. The intermediate layer contains the classes that implement the algorithms and features provided by Peabrain. Finally, the GUI layer encloses the classes related to graphic interfaces for collecting/showing from/to the user input data and results of the functionalities.

The integration of Peabrain in PIPE is depicted in Fig. 2. PIPE allows to be extended through modules that must implement the IModule interface. Note that the integration follows an open architecture, as upper layered classes communicate with non-immediate lower layered classes. Namely, Fig. 2 shows how the PIPE-data layer and Peabrain-data layer are related. The PetriNetModel class, which is a matrix representation of the current PN model in PNML (PIPE format), is created by each one of the Peabrain modules. Let us remark that our modules do not use PNML as data layer because the module algorithms work with the matrix representation as we are mainly solving LPPs.

\subsection{Plug-in architecture}

Peabrain was designed following also a plug-in architecture to facilitate its extension. This architecture makes easier to add more functionalities that make use of LP techniques on PNs. In fact, this is the major benefit of this architecture: It allows a rapid prototype implementation of any algorithm that deals with Petri nets and LP problems. Let us explain how Peabrain can be easily extended by an example.

Recall that to check whether a place is (structurally) implicit can be solved by an LPP (see LPP (1)). Suppose that a user wants to add this feature to Peabrain. The first thing to do is to create a class (termed as StructuralImplicitPlace) that extends the abstract class WellFormedLPP, and fill in the methods properly. Recall that the 


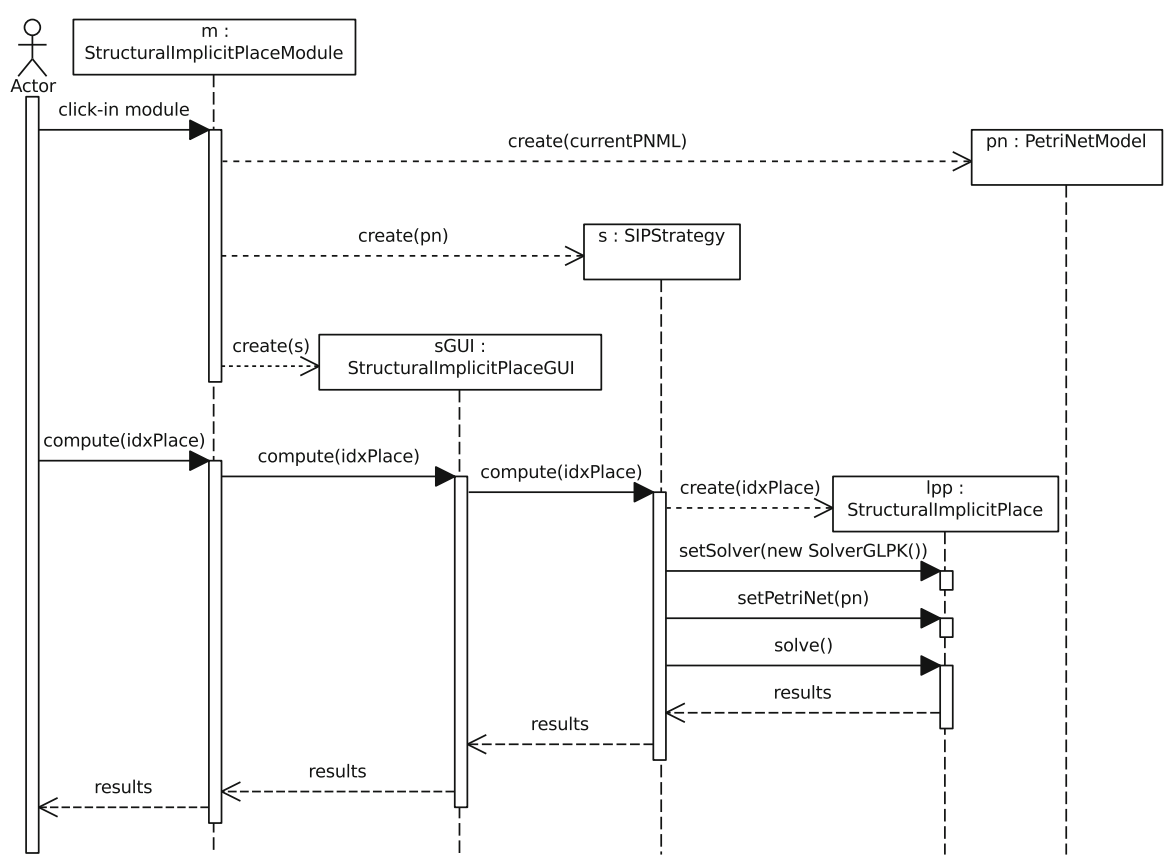

Fig. 3 UML Sequence Diagram for executing Structural Implicit Place module

Petri net is represented in matrix form by the PetriNetModel class, and the methods of StructuralImplicitPlace represent the LPP (1) variables, constraints, objective function, etc. After that, an intermediate layer class must be created, SIPStrategy, extending the Strategy abstract class. This class must properly create and communicate with the new class created (StructuralImplicitPlace). After creating it, it needs to fill in the arguments needed by StructuralImplicitPlace (if any) and then invoke to solve() method. Finally, a class should be created in the GUI layer by extending the PeabrainGUI abstract class (let us suppose this class is named as StructuralImplicitPlaceGUI). As last step, the integration with PIPE must be performed. Thus, a new module named StructuralImplicitPlaceModule implementing IModule and using PetriNetModel is created.

Figure 3 illustrates interactions between the user, PIPE and Peabrain when executing this new feature considering the above mentioned classes. Once the StructuralImplicitPlaceModule is instanced, it creates a PetriNetModel object, taking as parameter the current PNML displayed by PIPE. After that, it creates a SIPStrategy and a StructuralImplicitPlaceGUI, which take as input parameter the recently created PetriNetModel object. The GUI also collects the input data provided by the user (i.e. the index of place to check, idxPlace), and then whether such a place is structurally implicit is checked. The last release of Peabrain includes indeed this feature, but the intermediate and GUI layered classes are integrated into Structurallterator and StructuralBoundGUI, respectively, as it shares some characteristics with other structural properties (mainly, the input parameters can be collected using the same GUI). 


\subsection{Tool modules}

In the following, the Peabrain modules and what they provide are introduced.

Performance estimation It uses an iterative algorithm based on LP problems (Rodríguez and Júlvez 2010; Rodríguez et al. 2013) for computing upper throughput bounds. This iterative approach is applicable for some subclasses of Petri nets (namely, Marked Graphs Murata 1989 and Process Petri nets Tricas 2003).

Resource optimisation This module enacts an optimal distribution of resources in a shared-resource PN for a given budget and resource costs, trying to enhance the system performance as much as possible was implemented. The theory behind this method was formerly introduced in (Rodríguez et al. 2013). This method is currently applicable for Process Petri nets (Tricas 2003).

Visit ratio computation Section 3.1 describes how the vector of visit ratios $v$ of a PN, normalised for a transition $t \in T$ can be computed. This module computes whether the vector of visit ratios has a single solution, otherwise reports that there exists more than one solution, and it cannot be computed.

Linear bound This module encompasses several computations related to linear bounds, such as the computation of slowest p-semiflow (see LPP (6)) or the computation of lower(upper) throughput bounds (see LPP (7)) (Campos and Silva 1993). Recall that the PN structure needs to fulfil a set of conditions so that the computation of performance bounds has some sense. These conditions are: (i) the PN must be structurally live, (ii) structurally bounded, (iii) have a home state and (iv) its vector of visit ratios must have a unique solution. Our tool is limited to automatically check the latter property, as verifying some of these properties in general nets are NP-decidability problems (Esparza and Nielsen 1994). The latter conditions are also applicable to the computation of the slowest $\mathrm{p}$-semiflow of a PN since it is indeed an upper performance bound for the real system performance.

This module also enables to compute the lower and upper average marking bound for a place in a Petri net, using LPP (7) but changing the optimisation function as required.

Structural marking bound and structural enabling bound The structural marking of a place $p \in P$, and the structural enabling of a transition $t \in T$, can as well be computed by LP problems (Campos and Silva 1993). These LP problems are valid for any kind of PN.

Structural implicit places This module allows to compute the structural implicit places of a net and the initial marking such that these places become implicit and thus they can be removed from the net. Both features can be solved by using LPP, as previously explained (see Secti. 3.1).

This module has been used as example of how to extend the plug-in architecture of PeabraiN. The classes implementing these features have been highlighted (orange) in Fig. 1. Note that these new classes belong to data layer. As we have previously said, more logic has been added to some intermediate and GUI layer classes to make use of these features.

GSPN simulation analysis This module incorporates two different stochastic simulation methods for average throughput and average marking computation in SPNs: An exact (discrete) method using the first reaction method (Gillespie 1976), and an 
approximate (continuous) method using the Tau-Leaping method (Gillespie 2007, 2008).

The exact method is based on the Gillespie's stochastic simulation algorithm (SSA) (Gillespie 1976). It performs a set of replications of the simulation, and estimates the average throughput with a given confidence interval level and error accuracy. This algorithm uses a Monte Carlo procedure for numerically generating enabled transitions to be fired (Gillespie 1992).

Algorithm 1 shows the pseudo-code of the discrete SSA implemented. As input, it needs two parameters: The Petri net $\mathcal{S}$ to be simulated and the maximum system time $t_{\max }$ to be simulated. As output, it produces a vector $\chi$ of average throughput of transitions. As side product, the vector of average marking of places $\overline{\mathbf{m}}$ is also obtained.

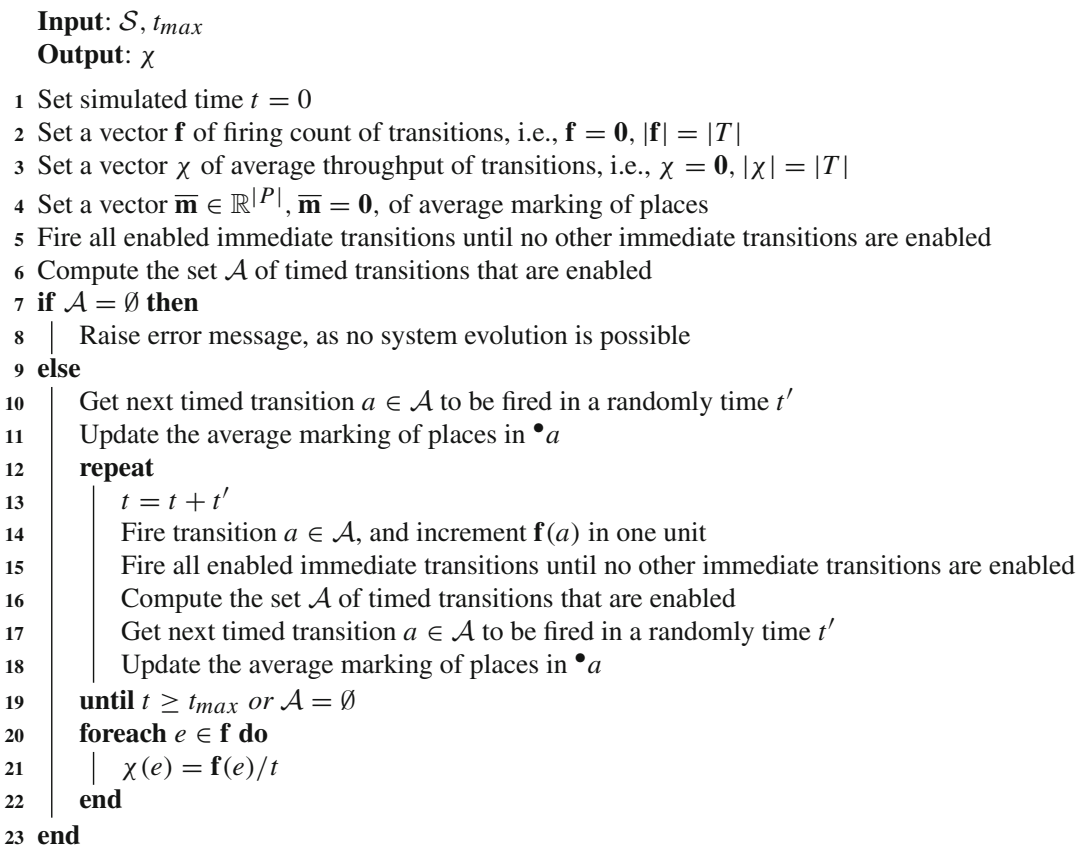

Algorithm 1: Discrete Stochastic Simulation Algorithm implemented in Peabrain.

Steps 1-4 initialise the parameters used in the algorithm, namely the simulated time, the vector of firing count of transitions, the vector of average throughput of transitions, and the vector of average marking of places. Then, all enabled immediate transitions are fired until no other immediate transition is enabled (step 5). Step 6 computes the set $\mathcal{A}$ of timed transitions that are enabled. Then, if no timed transition is enabled, an error message is reported indicating that the system cannot evolve, and suggests that initial marking might be revised. Otherwise, a transition $a \in \mathcal{A}$ is picked up for firing at time $t^{\prime}$ (step 10). Next step updates the average marking of places contained in $\bullet a$. This transition is selected by applying the standard inversion generating method of Monte 
Carlo theory (Gillespie 1992). Steps 12-19 are the simulation loop. The simulated time is incremented in $t^{\prime}$ units (step 13), and transition $a$ is fired and its firing count is incremented (step 14). Then, previous steps 5, 6, 10 and 11 are repeated. The iteration loop ends either when the maximum of simulated time is reached, or when there is no any timed transition to be fired. Finally, steps 20-22 compute the average throughput of each transition by dividing the number of firing between the total simulated time.

Peabrain provides other simulation method that implements Tau-Leaping method (Gillespie 2007, 2008), an approximate way of accelerating the SSA in which each time step advances the system through possibly many events at the same time. In terms of Petri nets, each step the enabled timed transitions are fired as much as possible, as indicated by a Poisson distribution.

Algorithm 2 introduces this simulation method. As input, it needs the Petri net to be simulated, and the maximum of simulated time. As output, it produces a vector $\chi$ of average throughput of transitions. As side product, the vector of average marking of places $\overline{\mathbf{m}}$ is also obtained. Note that both algorithms use the same input, and produce the same output.

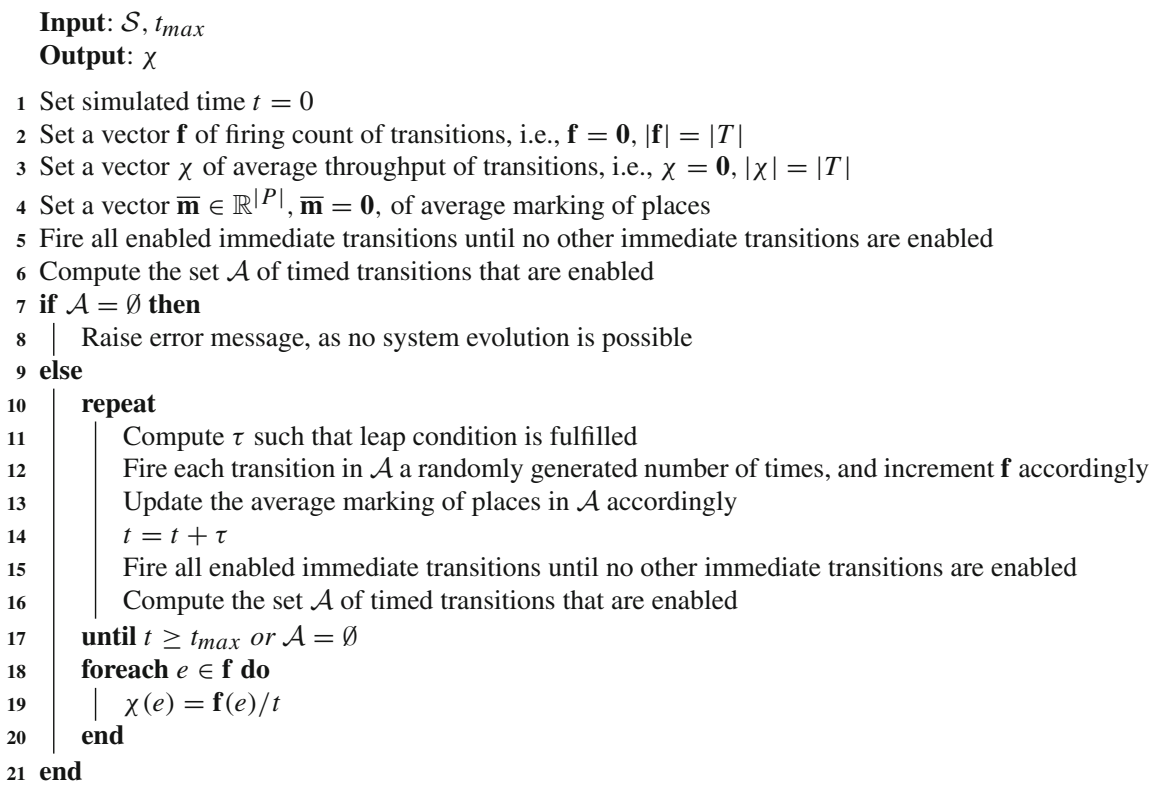

Algorithm 2: Tau-Leaping Stochastic Simulation Algorithm implemented in Peabrain.

Steps 1-9 are equivalent to the same steps at Algorithm 1. Both algorithms mainly differ in the iteration loop. Here, the iteration loop starts in step 11, where a time $\tau$ that fulfils the leap condition is computed. The leap condition ensures that such a $\tau$ is small enough that no propensity function changes by a significant amount (Gillespie 2007). Given a marking $\mathbf{m}$, such a value $\tau$ is computed as: 


$$
\tau=\min _{\forall a \in \mathcal{A}}\left(\frac{\max \left(\epsilon \cdot \sum_{\forall a \in A} \delta(a), 1\right)}{\left|\sum_{p \in \mathcal{B}_{a}} \mathbf{C}(p, a) \cdot \delta(a) \cdot \mathbf{e}(a)\right|}, \frac{\max \left(\epsilon \cdot \sum_{\forall a \in A} \delta(a), 1\right)^{2}}{\left|\sum_{p \in \mathcal{B}_{a}} \mathbf{C}(p, a)^{2} \cdot \delta(a) \cdot \mathbf{e}_{\mathbf{m}}(a)\right|}\right)
$$

where $\mathcal{B}_{a}=\left\{\bullet^{\bullet} a\right\} \bigcup\left\{a^{\bullet \bullet}\right\}, \delta(a)$ is the mean of the exponential firing time distribution associated to $a$, and $\mathbf{e}_{\mathbf{m}}(a)$ is the enabling degree of transition $a$.

After computing $\tau$, each enabled timed transition $a$ fires in $[t, t+\tau)$ a number of times $n$ which is a Poisson random variable with mean (and variance) $\delta(a) \cdot \mathbf{e}(a) \cdot \tau$, i.e., $n=\mathcal{P}(\delta(a) \cdot \mathbf{e}(a) \cdot \tau)$, and the vector of firing counts is accordingly incremented (step 12). Similarly, step 13 increments accordingly the average marking of places in $a, \forall a \in \mathcal{A}$. Then, step 14 increments the simulated time in $\tau$ units. As before, previous steps 5 and 6 are repeated. The iteration loop ends either when the simulated time reaches the maximum simulated time, or the set of enabled transitions is empty. As in Algorithm 1, the final steps (18-20) compute the average throughput of each transition.

Algorithm 2 outperforms Algorithm 1 for net systems where the population (i.e., the number of initial tokens) is large, as in each iteration step the enabled timed transitions are fired more than just one time. Of course, the simulation results produced by Algorithm 2 may be less exact than the ones computed by Algorithm 1. Note that enabled timed transitions are fired at a time, regardless these firings may enable subsequent immediate transitions. Thus, timed transitions could be fired multiple times at once which would change the net behaviour. This is an interesting issue that needs further study. We aim at studying the structure of nets whose behaviour may be influenced by this simulation method.

In the next section we compare the performance and accuracy of both algorithms in a case study. As future work, we aim at evaluating them with a large set of Petri nets.

\subsection{Extended features}

In this section, we summarise other features that Peabrain provides and are used by all modules. Namely, Peabrain allows to select the LP solver to be used and to execute all module using the command-line interface (CLI). The first feature could be used, for instance, to evaluate the performance of LP solvers in the computation of some Petri net properties; while the latter feature enables to batch executions without user interaction, and to concatenate the execution of different modules.

Linear programming solver selector A Peabrain Settings option has been added into PIPE to allow a user to select the LP solver to be used in LP computations. To this aim, we have followed a Model-View-Controller architectural pattern for the design of the solver selector window, which allows to the user to select the LP solver to be used by Peabrain modules. This window has been integrated into the PIPE menu, 


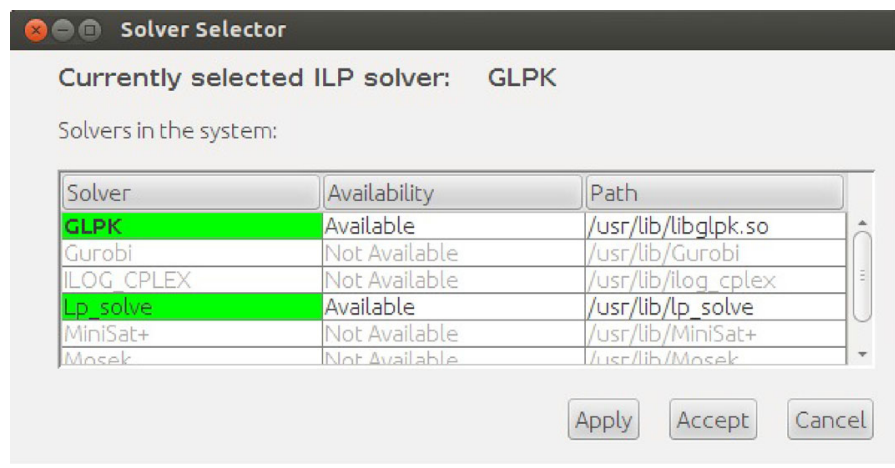

(a)

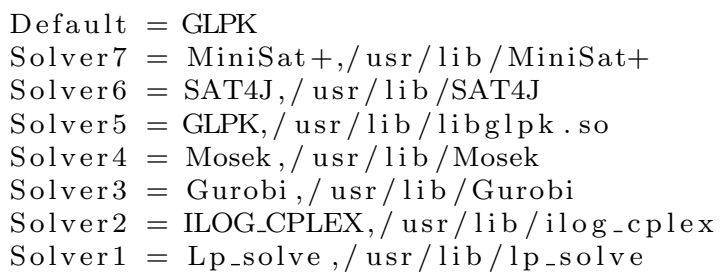

(b)

Fig. 4 a LP solver selector GUI and b configuration file to dynamically generate solver list

thus allowing an easily configuration from the main PIPE window. Besides, the LP solver list is dynamically generated and its content relies on a configuration file ${ }^{1}$ that can be easily modified (following a given syntax) by any user. This new GUI and a example of configuration file are given in Fig. 4a, b, respectively. We have also used the Singleton creational pattern to ensure a unique instance of the LP solver across all modules. This enhances the performance of Peabrain, as the same solver can be concurrently used by different modules, thus saving the LP loading penalty time.

Command-line interface. Peabrain also provides a way to execute any module via a CLI. This feature enables to batch computations in Petri nets without the need of user interaction, and even to easily integrate Peabrain modules and results with other tools.

Figure 5 depicts the UML-CD of Peabrain CLI modules. Note that the integration with Peabrain modules are done through module strategies (left-side classes of the figure). We have followed the same design rules as with Peabrain. Thus, the Facade structural pattern and a plug-in architecture have been used: When a new module needs CLI support, a developer only needs to extend PeabraiNCLI class and fill in the required methods properly. Moreover, this new functionality was designed under the idea of minimising as much as possible the modification of current code when adding CLI support for other modules. Therefore, the PipeCLI, which is the one currently

\footnotetext{
1 Named ".solverselector.conf", and located at the home folder of the user.
} 


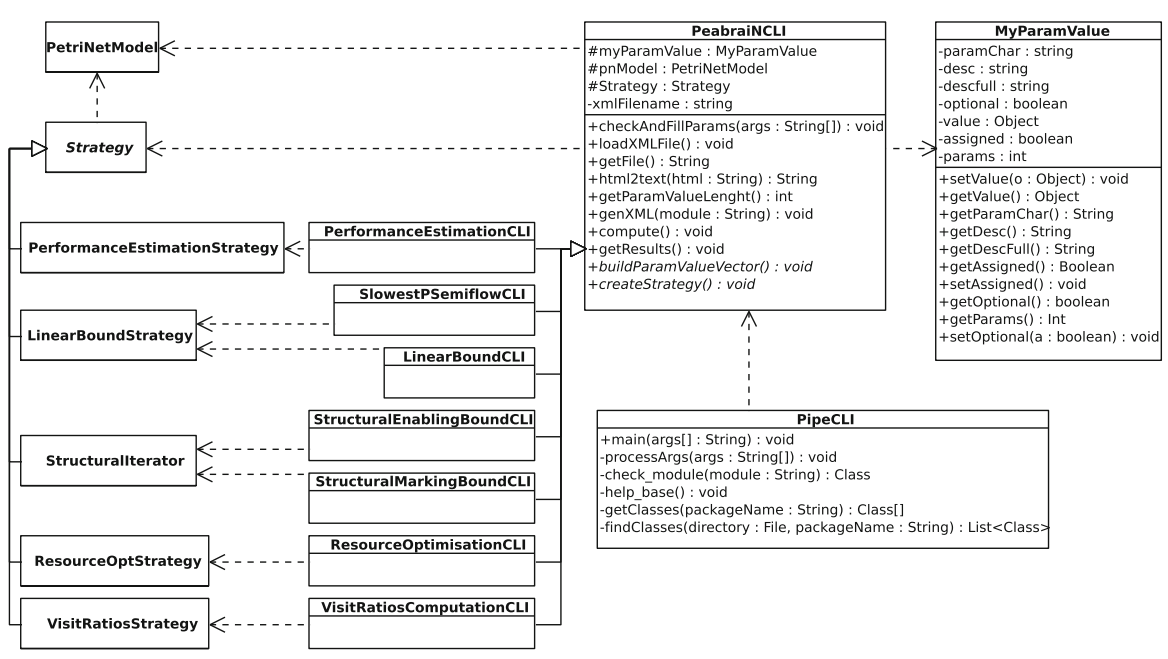

Fig. 5 UML Class diagram of Peabrain command-line interface

executed by a user when executing Peabrain through a console, automatically loads the modules that can be executed by checking a predefined CLI module path.

The support of these dynamic CLI modules rises other dynamic features, such as the dynamic generation of help messages, which report about the usage of each module with its corresponding module parameters. To this aim, the parameters are collected by the MyParamValue class, which defines a set of methods and properties for input parameters. Recall that a module has some mandatory (and common) parameters, such as the PNML file and the LP solver to be used by the module, and other specific parameters, such as the identification label of a place or a transition in the case of structural marking bound and structural enabling bound modules, respectively.

\subsection{Tool remarks}

In this last part, we summarise the kind of Petri nets where the methods provided by Peabrain can be applied. Exact simulation methods are valid to any kind of Petri net subclass (e.g., a simple net). However, approximate simulation methods are only applicable to nets where only immediate transitions are in conflict. Besides, this simulation method is recommended for nets with a high number of tokens since accuracy error is higher when population is low. Similarly, lower/upper throughput (marking) bound computation can be done in any kind of Petri net using the LPP (7). Iterative upper throughput bound algorithms, and resource optimisation, are valid for Petri nets where the vector of visit ratios is computable. Likewise, computing slowest p-semiflow using LPP 6 is only possible when the vector of visit ratios is known. Recall that the vector of visit ratios is easily computable when it depends only on the net structure and on the routing rates. Otherwise, the reachability set of a net is needed to compute it. 
Tool availability Peabrain is released under GNU GPL version 3 license, and further information about tool requirements and installation steps, tool binaries and sources can be found in the project web page http://webdiis.unizar.es/GISED/? $\mathrm{q}=$ tool/peabrain.

\section{Case study: a building CCTV system}

In this section, we study the performance of a Building Closed Circuit TV System (CCTV), inspired by the system introduced in (Petriu and Woodside 2003; Woodside et al. 2005). The CCTV collects video images from a set of cameras, stores them for forensics analysis if needed, and analyses them in real-time to report suspicious behaviours. The system is first modelled with UML and annotated with MARTE (OMG 2011a) profile, and then converted to a Petri net (PN). Lastly, its performance is estimated using upper throughput bound computation under different scenarios. We also simulate the system with PeabraiN and other existing PN tools to validate the results.

\subsection{System description}

Figure 6 depicts the UML deployment diagram (UML-DD) of CCTV, including the hardware resources (depicted as cubes) and their network links (arrows between cubes, or cubes in the case of intranets). The architecture of the CCTV is composed by a database server and an application server, both connected through an intranet. The database server deploys a component, DatabaseManager, in charge of interacting with the database, represented as an storage resource (HardDisk). The application server embraces three different elements: The access controller, the video acquisition and the alarm controller. The video acquisition controller is composed by four different resources: VideoController, AcquireProc, StoreProc y BufferManager. All resources are annotated with MARTE (OMG 2011a) to express its multiplicity (resource stereotype, and resmult tagged value). We assume that the system is able to run in parallel with several instances of each resource (but the VideoController), which outperforms a non-parallel execution.

In this paper, we focus on the UML Sequence Diagram (UML-SD) of acquire/store video scenario depicted in Fig. 7. The VideoController iterates acquiring and storing video frames for each camera deployed in the system. The number of cameras to iterate has been set to a variable $\$ n C$ ameras for sensitive analysis. Each image frame is processed by an instance of AcquireProc resource. This resource allocates a memory buffer to process the image. The memory buffer handling is performed by an instance of BufferManager resource. Once the memory buffer is allocated, the image frame is transferred over the network (represented by an external operation with a throughput depending on the number of packets $\$ n P$ transmitted) and sent to StoreProc resource to be stored into the database. An instance of StoreProc resource gets the image and writes it into the database. After that, it analyses the image and raises an alarm when some suspicious behaviour is detected. The image analysis can be carried out using a behavioural profiling based on multiple observations method to achieve a $91 \%$ of success (Bouma et al. 2013). Suspicious behaviour rate has set to \$ss Rate input 


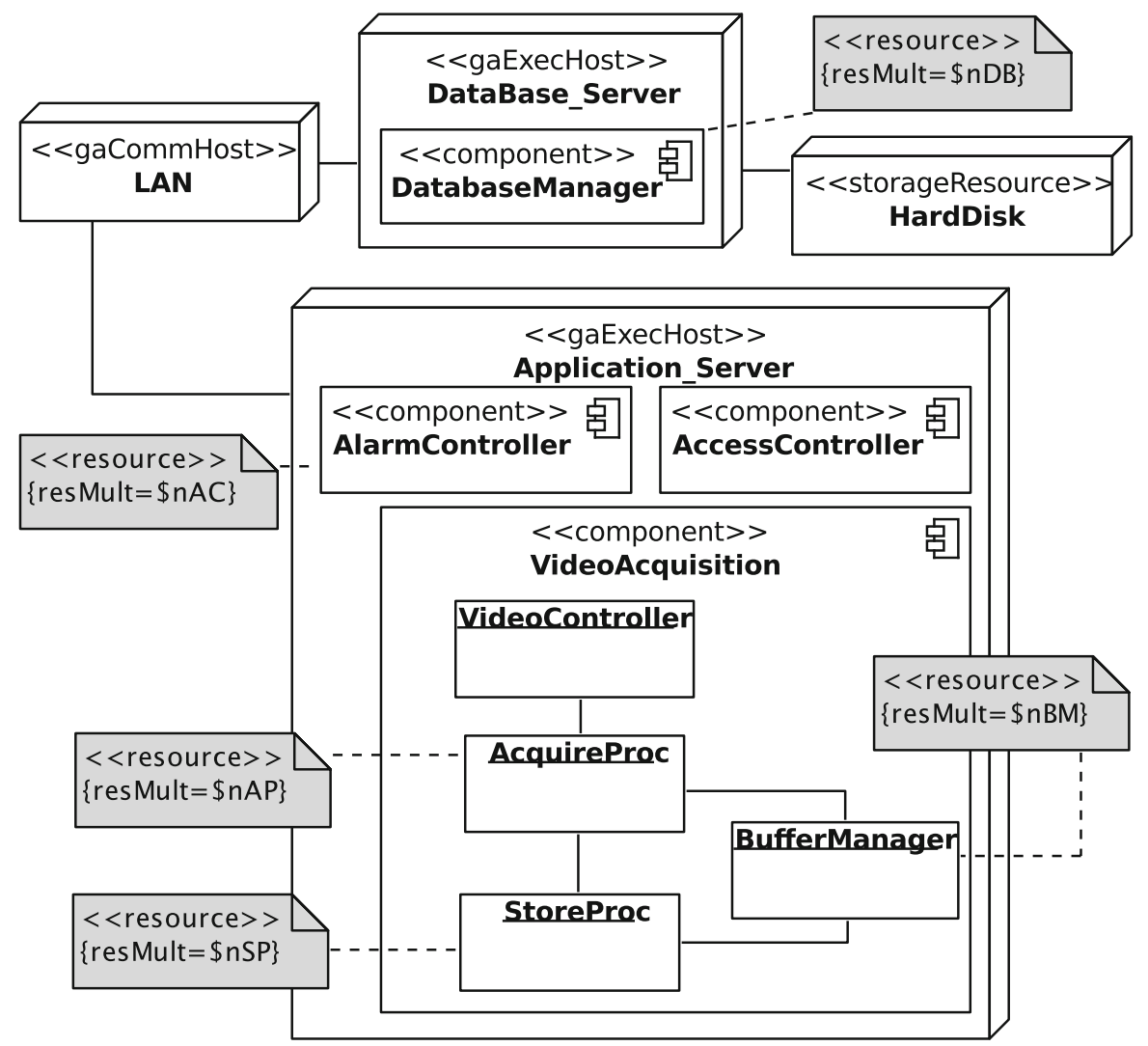

Fig. 6 CCTV UML deployment diagram (annotated with MARTE)

parameter, and will be used for sensitive analysis. Once the image has been analysed, the previous acquired memory buffer is released, and image processing for the camera is finished.

The acquire (release) of a resource has been indicated through the gaAcqStep (gaRelstep) stereotype (see MARTE annotations in Fig. 7), also denoting the number of resources acquired (released). To avoid cluttering we only show the first acquire (release) of resource AcquireProc. The rest of resources are annotated in the same way. Activities are annotated with gastep stereotype to specify how long takes, on average, each activity using the tagged-value hostDemand.

Description as a Petri net Figure 8 shows the PN obtained from the transformation of the UML-SD depicted in Fig. 7. The transformation from UML to PN is documented in (Distefano et al. 2011), and can be carried out by several tools, such as ArgoPN (Delatour and de Lamotte 2003), ArgoPerformance (Distefano et al. 2011) or ArgoSPE (Gómez-Martínez and Merseguer 2006) (see Sect. 2). In this paper, ArgoSPE tool $^{2}$ has been chosen since the output format is compatible with GreatSPN tool (Baarir

2 Available at https://argospe.tigris.org. 


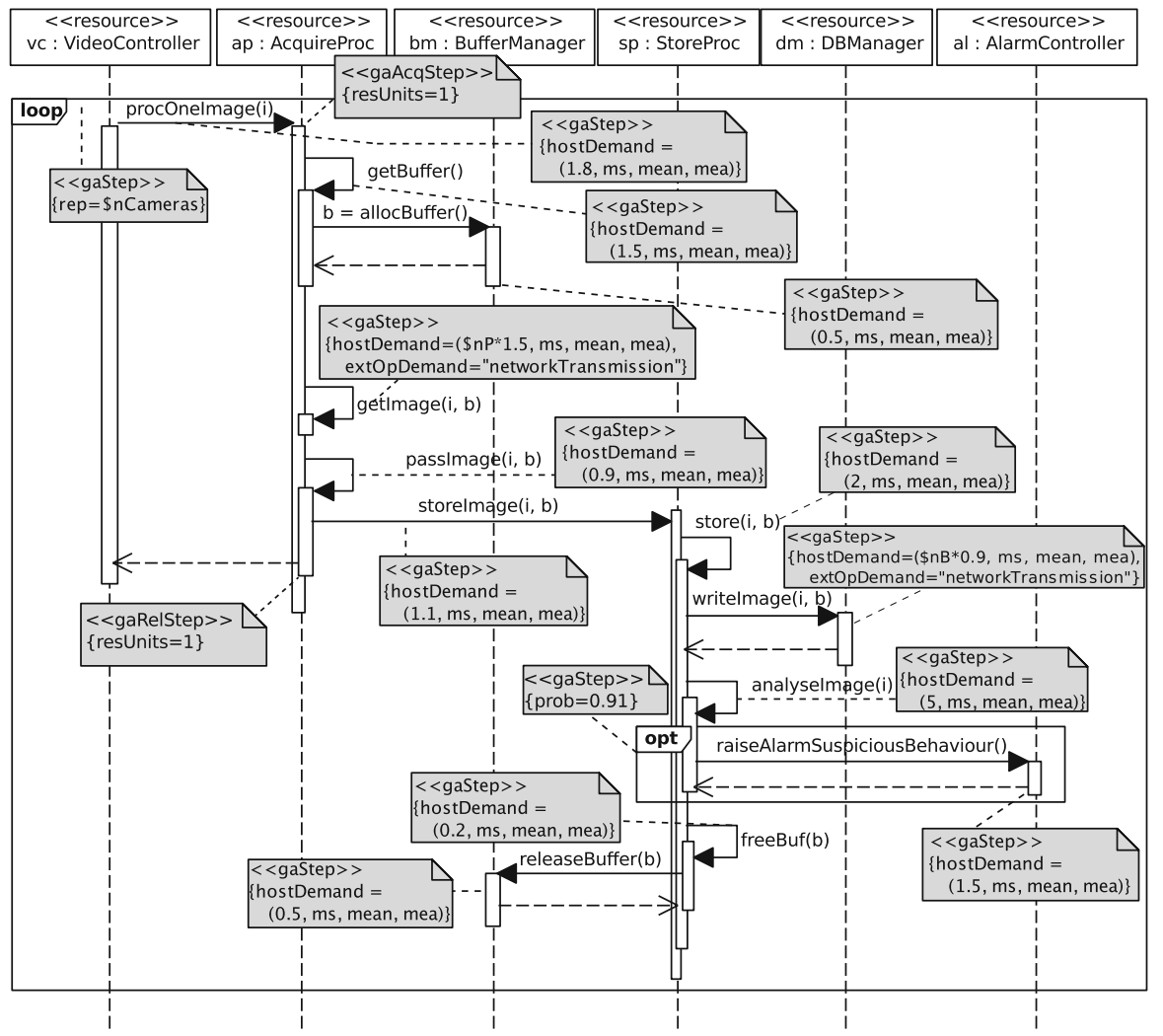

Fig. 7 CCTV acquire/store video scenario

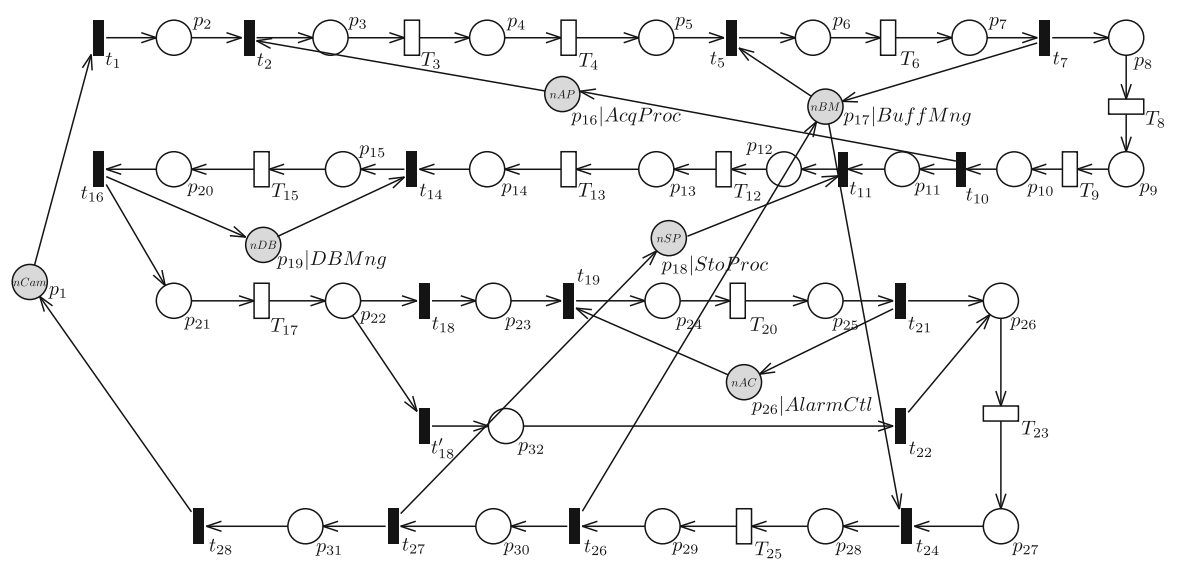

Fig. 8 Petri net of the CCTV. Resource places are depicted in light grey

et al. 2009), a PN tool later used for simulate the system. A parser from GreatSPN input net format to Peabrain has also been implemented. Note that ArgoSPE is useful in this context since software engineers usually work with UML diagrams and 
Table 1 CCTV experiment settings

\begin{tabular}{lll}
\hline Transition & Activity & Value(s) \\
\hline $\begin{array}{ll}\text { (a) Delay of net transitions } \\
T_{3}\end{array}$ & procOneImage & $1.8 \mathrm{~ms}$ \\
$T_{4}$ & getBuffer & $1.5 \mathrm{~ms}$ \\
$T_{6}$ & allocBuffer & $0.5 \mathrm{~ms}$ \\
$T_{8}$ & getImage & $12 \mathrm{~ms}$ (assuming $\$ n P=8)$ \\
$T_{9}$ & passImage & $0.9 \mathrm{~ms}$ \\
$T_{12}$ & storeImage & $1.1 \mathrm{~ms}$ \\
$T_{13}$ & store & $2 \mathrm{~ms}$ \\
$T_{15}$ & writeImage & $7.2 \mathrm{~ms}($ assuming $\$ n B=8)$ \\
$T_{17}$ & analyseImage & $5 \mathrm{~ms}$ \\
$t_{18}$ & \$suspiciousProb & 0.91 \\
$t_{18}^{\prime}$ & (1 - \$suspiciousRate) & 0.09 \\
$T_{20}$ & raiseAlarm & $2 \mathrm{~ms}$ \\
$T_{23}$ & freeBuff & $0.2 \mathrm{~ms}$ \\
$T_{25}$ & releaseBuff & $0.5 \mathrm{~ms}$ \\
\hline Place & Parameter & Value(s) \\
\hline & &
\end{tabular}

(b) Initial marking of net places

$\begin{array}{lll}p_{1} & \text { No. of cameras } & \{50,100,150\} \\ p_{16} & \text { Acquire process instances } & \{10,20,30\} \\ p_{17} & \text { Buffer manager instances } & \{10,20,30\} \\ p_{18} & \text { Store process instances } & \{10,20,30\} \\ p_{19} & \text { Database manager instances } & 5 \\ p_{26} & \text { Alarm controller instances } & 2\end{array}$

this tool transforms them to PNs. Nevertheless, any of the other tools could be used in conjunction with PeabraiN to obtain a PN model from a UML model. Note that any system transformed to Petri net could be analysable with PeabraiN. Of course, depending on the net structure some features may not apply (see Sect. 4.5).

Each resource annotated in Fig. 6 is represented by a (light-grey highlighted) place in the PN: $p_{1}$ (number of cameras), $p_{16}$ (acquire process), $p_{17}$ (buffer manager), $p_{18}$ (store process) and $p_{19}$ (database manager), and $p_{33}$ (alarm controller). Table 1 summarises the number of instances of each resource, since they will be represented by tokens in the respective places.

The acquire (release) of a resource is transformed into an immediate transition with an input (output) arc. For example, transition $t_{2} 3$ represents the acquire of the Acquire Process, while $t_{10}$ represents the release of such a resource. The activities, self-messages in Fig. 7, are transformed into an exponential transition in the PN with its corresponding duration (given in Table 1).

In the sequel, we analyse the properties of the PN obtained after transformation from the UML-SD of Fig. 7. The net is composed of 33 places and 29 transitions. In fact, the PN is a process Petri net (Tricas 2003), a subclass of PN where 
the analysis methods introduced in (Rodríguez et al. 2013) to compute improved upper throughput bounds and resource optimisation apply. The net has six minimal p-semiflows: $\mathbf{y}_{1}=\left\{p_{1-15}, p_{20-32}\right\}, \mathbf{y}_{2}=\left\{p_{3-10}, p_{16}\right\}, \mathbf{y}_{3}=\left\{p_{6}, p_{7}, p_{17}, p_{28}, p_{29}\right\}$, $\mathbf{y}_{4}=\left\{p_{12-15}, p_{18}, p_{20-30}, p_{32}\right\}, \mathbf{y}_{5}=\left\{p_{15}, p_{19}, p_{20}\right\}, \mathbf{y}_{6}=\left\{p_{24-26}\right\}$. For the sake of readability, we have shorten and grouped the place names. All places are covered by some p-semiflow, therefore the net is bounded. Similarly, the net has two minimal t-semiflows: $\mathbf{x}_{1}=\left\{t_{1-21}, t_{23-28}\right\}$ and $\mathbf{x}_{2}=\left\{t_{1-28}, t_{18}^{\prime}\right\}$. All transitions are covered by some t-semiflow, there it might be bounded and live. Transitions $t_{18}, t_{18}^{\prime}$, are in an extended free-choice conflict, while transitions $t_{5}, t_{24}$, are in structural (but not freechoice) conflict. When both transitions are in an effective conflict for a given marking $\overline{\mathbf{m}}$, the transition to be fired is randomly chosen.

We compute the structural implicit places using the new feature of Peabrain. The net has four implicit places, which match with the places representing resources (but the number of cameras) within the system: $p_{16}$ (acquire process), $p_{17}$ (buffer manager), $p_{18}$ (store process) and $p_{19}$ (database manager), and $p_{33}$ (alarm controller). In fact, the initial marking for each one of these places such that they become structurally implicit is equal to the initial marking of $p_{1}$ (number of cameras in the system). That is, if the initial marking of the former places are greater than or equal to the initial parking of $p_{1}$, then they can be removed without effect to the behaviour of the Petri net.

\subsection{Experiments and discussion}

In the sequel, we validate PeabraiN's new features by evaluating the CCTV under different workloads. We set the number of resources as expressed in Table 1: The number of cameras will vary between 50, 100 and 150, while the number of resource instances in the application server varies between 10, 20 and 30 (but the alarm controller, which remains equal to 2). Similarly, the database manager instances is set to 5 .

The experiments have been run in a machine with Intel Pentium 4 CPU $3.60 \mathrm{GHz}$ $2048 \mathrm{KiB}$ cache, $3 \mathrm{GiB}$ RAM $533 \mathrm{MHz}$, running a 32-bit Linux distribution. The net depicted in Fig. 8 has been simulated with the two simulation methods provided by Peabrain (exact, approximate). The set of experiments has been executed with two different confidence level and accuracy configurations: 95-5, and 99-1\%.

Tables 2 and 3 summarise the throughput and execution time results of the set of experiments performed, respectively. Throughput results are taken from the throughput of transition $t_{1}$. The first column indicates the number of cameras, while the second the number of other resource instances in the application server. Then, the results given by PeabraiN using both simulation methods, as well as its execution time (in seconds), is shown. Lastly, the percentage of approximate simulation result with respect to exact simulation result is shown.

The results of Table 2 show that approximate simulation method performs better when the number of tokens is high. In fact, the results are almost the same with the most populated net. It also remarkable that the simulation parameters (confidence level and accuracy) do not a have a big impact in the results. In all cases, the difference remains at $\pm 0.5 \%$. 
Table 2 CCTV experiment: throughput results

\begin{tabular}{llll}
\hline $\begin{array}{l}\text { No. of } \\
\text { cameras }\end{array}$ & $\begin{array}{l}\text { Other } \\
\text { resource } \\
\text { instances }\end{array}$ & $\begin{array}{l}\text { Peabrain } \\
\text { (exact) }\end{array}$ & $\begin{array}{l}\text { Peabrain } \\
\text { (approximate) }\end{array}$
\end{tabular}

Confidence interval $95 \%$, accuracy $5 \%$

\begin{tabular}{|c|c|c|c|c|}
\hline \multirow[t]{3}{*}{50} & 10 & 0.5788341 & 0.6130577 & 5.91 \\
\hline & 20 & 0.6933366 & 0.6956743 & 0.34 \\
\hline & 30 & 0.6942851 & 0.69478 & 0.07 \\
\hline \multirow[t]{3}{*}{100} & 10 & 0.5818307 & 0.6154501 & 5.78 \\
\hline & 20 & 0.6981312 & 0.69637 & -0.25 \\
\hline & 30 & 0.6972403 & 0.6957046 & -0.22 \\
\hline \multirow[t]{3}{*}{150} & 10 & 0.5839596 & 0.6169496 & 5.65 \\
\hline & 20 & 0.6973815 & 0.6995541 & 0.31 \\
\hline & 30 & 0.6958464 & 0.6958727 & 0.00 \\
\hline \multicolumn{5}{|c|}{ Confidence interval 99, accuracy $1 \%$} \\
\hline \multirow[t]{3}{*}{50} & 10 & 0.5799806 & 0.612705 & 5.64 \\
\hline & 20 & 0.6967369 & 0.6958847 & -0.12 \\
\hline & 30 & 0.6952727 & 0.6961634 & 0.13 \\
\hline \multirow[t]{3}{*}{100} & 10 & 0.5812165 & 0.6148568 & 5.79 \\
\hline & 20 & 0.6963155 & 0.6929288 & -0.49 \\
\hline & 30 & 0.6963199 & 0.6939515 & -0.34 \\
\hline \multirow[t]{3}{*}{150} & 10 & 0.5846464 & 0.6176673 & 5.65 \\
\hline & 20 & 0.6971197 & 0.6964626 & -0.09 \\
\hline & 30 & 0.6963669 & 0.6956921 & -0.10 \\
\hline
\end{tabular}

Similarly, the execution of approximate simulation method clearly outperforms the execution of exact simulation method. Table 3 shows that approximate simulation method executes near to half a time the execution time of exact simulation method, for all cases under study.

In brief, the approximate simulation method is recommended for highly populated nets (i.e., Petri nets with a huge number of tokens). About the simulation parameters, a high confidence level and low accuracy error are recommended since the difference on execution time when changing them is negligible.

Let us compute the upper throughput bound of the most populated net in the experiments (that is, 150 cameras and 30 other resources). The slowest p-semiflow is $\mathbf{y}_{5}=\left\{p_{15}, p_{19}, p_{20}\right\}$, with a throughput of 0.694445 . The computation of this bound takes $0.2830 \mathrm{~s}$. Note that this upper throughput bound is almost identical to the simulation result, but the execution time is less than half a second, instead of $220.4 \mathrm{~s}$ (or 223.38 s, with confidence level $99 \%$, accuracy error $1 \%$ ).

The slowest p-semiflow is also given feedback about what is the most constraining resource/part in the system. Thus, whether a system designer would need to optimise this system, s/he has two choices: either to increment the number of instances of database manager (since the place representing such a resource is contained in the support of $\mathbf{y}_{5}$ ), or to improve the function writeImage represented by transition $T_{15}$ (see Table 1). 
Table 3 CCTV experiment: execution time results (in seconds)

\begin{tabular}{|c|c|c|c|c|}
\hline $\begin{array}{l}\text { No. of } \\
\text { cameras }\end{array}$ & $\begin{array}{l}\text { Other resource } \\
\text { instances }\end{array}$ & $\begin{array}{l}\text { Peabrain } \\
\text { (exact) }\end{array}$ & $\begin{array}{l}\text { Peabrain } \\
\text { (approximate) }\end{array}$ & $\%$ \\
\hline \multicolumn{5}{|c|}{ Confidence interval $95 \%$, accuracy $5 \%$} \\
\hline \multirow[t]{3}{*}{50} & 10 & 206.529 & 98.692 & -52.21 \\
\hline & 20 & 216.059 & 109.268 & -49.43 \\
\hline & 30 & 220.462 & 114.761 & -47.95 \\
\hline \multirow[t]{3}{*}{100} & 10 & 182.443 & 99.581 & -45.42 \\
\hline & 20 & 219.214 & 108.902 & -50.32 \\
\hline & 30 & 220.079 & 109.632 & -50.19 \\
\hline \multirow[t]{3}{*}{150} & 10 & 181.053 & 101.071 & -44.18 \\
\hline & 20 & 218.096 & 110.519 & -49.33 \\
\hline & 30 & 220.4 & 110.857 & -49.70 \\
\hline \multicolumn{5}{|c|}{ Confidence interval $99 \%$, accuracy $1 \%$} \\
\hline \multirow[t]{3}{*}{50} & 10 & 179.754 & 98.978 & -44.94 \\
\hline & 20 & 220.374 & 114.931 & -47.85 \\
\hline & 30 & 218.702 & 113.891 & -47.92 \\
\hline \multirow[t]{3}{*}{100} & 10 & 181.305 & 101.368 & -44.09 \\
\hline & 20 & 215.844 & 108.217 & -49.86 \\
\hline & 30 & 217.812 & 109.141 & -49.89 \\
\hline \multirow[t]{3}{*}{150} & 10 & 182.096 & 100.06 & -45.05 \\
\hline & 20 & 219.847 & 111.187 & -49.43 \\
\hline & 30 & 223.386 & 110.701 & -50.44 \\
\hline
\end{tabular}

Therefore, upper throughput bounds computation provides to software designers rapid information about what functions and resources can be optimised in order to improve system performance.

\section{Conclusions and future work}

Software systems modelling with UML, standard de facto in the software development industry, can be enriched with UML profiles such as MARTE to specify performance data (e.g., duration of activities, transmissions, etc.) within UML models. These annotated UML models can be transformed to formal models to enhance the analysis capabilities. In this paper, we consider Petri nets as formal model obtained from UML models enriched with MARTE annotations. Existing tools for performance (or throughput) evaluation in Petri nets rely either on the exploration of the whole state space, or either on simulating the net. However, these methods are unfeasible for large systems either because of the well-known state-space explosion appears, or simulation does not converge in a reasonable time. To overcome these issues, upper throughput bounds can be computed. This paper introduces Peabrain, a Petri net tool that enables, among other features, the computation of upper (and lower) throughput bounds. 
Peabrain is a collection of PIPE-modules to simulate and compute several Petri net properties that can be compute using Linear Programming problems. Some of these properties are, for instance, upper (and lower) throughput bounds, slowest psemiflow or structural marking (and enabling). Peabrain also enables to compute structural implicit places of a net, and lower and upper average marking bound for places. Besides, it allows to select the Linear Programming solver to be used, and incorporates a CLI to easily batch computations and collect results. Apart from a exact simulation method, it implements an approximate method to simulate Stochastic Petri nets using the Tau-Leaping method.

This paper introduces the plug-in architecture of Peabrain and how it can be easily extended, as well as the modules and features provided by the tool. By means of a case study that models a building closed circuit TV system we also show the benefit of using upper throughput bounds to evaluate the performance in large systems where the exact performance computation becomes unfeasible, in terms of execution time. In summary, the usage of upper throughput bounds provides a software designer in a fast way information about what are the slowest methods in the system, in which initial improvement efforts should be targeted to obtain a better system performance.

As future work, we plan to integrate PeabraiN with UML CASE tools. This integration brings several benefits into the foreground: First, formal methods can be automatically adopted in the design and analysis of UML models; and second, it easily provides feedback to the software engineers about what the most constraining resources/slowest activities in the system are, and then more effort should be done to optimise them. We also aim at extending the tool with more features related to Petri nets. We are at the moment implementing a web service using AJAX and PHP for remotely executing Peabrain.

Acknowledgements The author would like to thank Iván Pamplona, an undergraduate student who helped in the implementation of PeabraiN enhancements. This work was partially supported by the EU Horizon 2020 research and innovation programme under Grant Agreement No. 644869 (DICE) and by the Spanish MICINN project CyCriSec (TIN2014-58457-R).

\section{References}

Baarir, S., Beccuti, M., Cerotti, D., De Pierro, M., Donatelli, S., Franceschinis, G.: The GreatSPN tool: recent enhancements. SIGMETRICS Perform. Eval. Rev. 36(4), 4-9 (2009)

Berardinelli, L., Bernardi, S., Cortellessa, V., Merseguer, J.: UML profiles for non-functional properties at work: analyzing reliability, availability and performance. In: Boskovic, M., Gasevic, D., Pahl, C., Schatz, B. (eds.) 2nd International Workshop on Non-functional System Properties in Domain Specific Modeling Languages (NFPinDSML), CEUR, CEUR, Denver, vol. 553 (2009)

Bernardi, S., Merseguer, J.: Performance evaluation of UML design with stochastic well-formed nets. J. Syst. Softw. 80(11), 1843-1865 (2007)

Bernardi, S., Merseguer, J., Petriu, D.: A dependability profile within MARTE. J. Softw. Syst. Model. 10(3), 313-336 (2011)

Bonet, P., Lladó, C., Puigjaner, R., Knottenbelt, W.: PIPE v2.5: a Petri net tool for performance modelling. In: Proceedings of the 23rd Latin American Conference on Informatics (CLEI), Costa Rica (2007)

Bonet, P., Lladó, C.M.: Importing PMIF models into PIPE2 using M2M transformation. In: Proceedings of the 3rd ACM/SPEC International Conference on Performance Engineering (ICPE). ACM, New York, pp. 245-246 (2012) 
Bouma, H., Vogels, J., Aarts, O., Kruszynski, C., Wijn, R., Burghouts, G.: Behavioral profiling in CCTV cameras by combining multiple subtle suspicious observations of different surveillance operators. In: SPIE Defense, Security, and Sensing, International Society for Optics and Photonics, p. 87451A (2013)

Campos, J., Silva, M.: Structural techniques and performance bounds of stochastic Petri net models. Lect. Notes Comput. Sci. 609, 352-391 (1992)

Campos, J., Silva, M.: Embedded product-form queueing networks and the improvement of performance bounds for Petri net systems. Perform. Eval. 18(1), 3-19 (1993)

Chiola, G., Anglano, C., Campos, J., Colom, J., Silva, M.: Operational analysis of timed Petri nets and application to the computation of performance bounds. In: Proceedings of the 5th International Workshop on Petri Nets and Performance Models (PNPM), IEEE Computer Society Press, Toulouse, pp. 128-137 (1993)

Delatour, J., de Lamotte, F.: ArgoPN: a CASE tool merging UML and Petri nets. In: Proceedings of the 3rd International Workshop on New Developments in Digital Libraries (NDDL) and the 1st International Workshop on Validation and Verification of Software for Enterprise Information Systems (VVEIS), pp. 94-102 (2003)

Distefano, S., Scarpa, M., Puliafito, A.: From UML to Petri nets: the PCM-based methodology. IEEE Trans. Softw. Eng. 37(1), 65-79 (2011)

Esparza, J., Nielsen, M.: Decidability issues for Petri nets—a survey. Bull. EATCS 52, 244-262 (1994)

Fecher, H., Schönborn, J., Kyas, M., Roever, W.P.: 29 New unclarities in the semantics of UML 2.0 state machines. In: Lau, K.K., Banach, R. (eds.) Formal Methods and Software Engineering. Lecture Notes in Computer Science, pp. 52-65. Springer, Berlin (2005)

Florin, G., Natkin, S.: Necessary and sufficient ergodicity condition for open synchronized queueing networks. IEEE Trans. Softw. Eng. 15(4), 367-380 (1989)

Garcia-Valles, F., Colom, J.: Implicit places in net systems. In: Proceedings of the 8th International Workshop on Petri Nets and Performance Models, pp. 104-113 (1999)

Gillespie, D.: Markov Processes: An Introduction for Physical Scientists. Academic Press, New York (1992)

Gillespie, D.T.: A general method for numerically simulating the stochastic time evolution of coupled chemical reactions. J. Comput. Phys. 22(4), 403-434 (1976)

Gillespie, D.T.: Stochastic simulation of chemical kinetics. Annu. Rev. Phys. Chem. 58, 35-55 (2007)

Gillespie, D.T.: Simulation methods in systems biology. In: Formal Methods for Computational Systems Biology, Lecture Notes in Computer Science, vol. 5016, Springer, Berlin, pp. 125-167 (2008)

Gómez-Martínez, E., Merseguer, J.: argospe: model-based software performance engineering. In: International Conference of Application and Theory of Petri Nets, pp. 401-410 (2006)

Hillah, L.M., Kindler, E., Kordon, F., Petrucci, L., Tréves, N.: A primer on the Petri net markup language and ISO/IEC 15909-2. Petri Net Newsl. 76, 9-28 (2009)

JavaILP: Java interface to ILP solvers library. http://javailp.sourceforge.net/ (2013)

Koch, M., Parisi-Presicce, F.: UML specification of access control policies and their formal verification. Softw. Syst. Model. 5(4), 429-447 (2006)

Lagarde, F., Espinoza, H., Terrier, F., Gérard, S.: Improving UML profile design practices by leveraging conceptual domain models. In: Proceedings of the 22nd IEEE/ACM International Conference on Automated Software Engineering, ACM, New York, ASE'07, pp. 445-448 (2007)

Liu, Z.: Performance bounds for stochastic timed Petri nets. In: Proceedings of the 16th ICATPN, SpringerVerlag, Berlin, pp. 316-334 (1995)

Lladó, C.M., Harrison, P.G.: A PMIF with Petri net building blocks. In: Proceedings of the 2nd ACM/SPEC International Conference on Performance Engineering (ICPE), ACM, New York, pp. 103-108 (2011)

López-Grao, J.P., Merseguer, J., Campos, J.: From UML activity diagrams to stochastic Petri nets: application to software performance engineering. In: Proceedings of the 4th International Workshop on Software and Performance (WOSP), ACM, New York, pp. 25-36 (2004)

Merseguer, J., Campos, J., Bernardi, S., Donatelli, S.: A compositional semantics for UML state machines aimed at performance evaluation. In: Proceedings of the 6th International Workshop on Discrete Event Systems (WODES), IEEE Computer Society, Washington, DC, WODES '02, pp. 295-302 (2002)

Molloy, M.: Performance analysis using stochastic Petri nets. IEEE Trans. Comput. C-31(9), 913-917 (1982)

Murata, T.: Petri nets: properties, analysis and applications. In: Proceedings of the IEEE, vol. 77, pp. 541-580 (1989)

NIST: JAMA: A Java matrix package. http://math.nist.gov/javanumerics/jama/ (2012) 
OMG: A UML profile for modeling and analysis of real time embedded systems (MARTE). Object Management Group, document formal/11-06-02 (2011a)

OMG: Unified modelling language: superstructure. Object Management Group, version 2.4, formal/11-0805 (2011b)

Petriu, D., Woodside, C.: Performance analysis with UML. In: Lavagno, L., Martin, G., Selic, B. (eds.) UML for Real, pp. 221-240. Springer, Heidelberg (2003)

Randimbivololona, F.: Orientations in verification engineering of avionics software. In: Informatics, Lecture Notes in Computer Science, vol. 2000. Springer, Berlin, pp. 131-137 (2001)

Rodríguez, R.J., Júlvez, J.: Accurate performance estimation for stochastic marked graphs by bottleneck regrowing. In: Proceedings of the 7th European Performance Engineering Workshop (EPEW), Lecture Notes in Computer Science, vol. 6342. Springer, Berlin, pp. 175-190 (2010)

Rodríguez, R.J., Merseguer, J., Bernardi, S.: Modelling and analysing resilience as a security issue within UML. In: Proceedings of the 2nd International Workshop on Software Engineering for Resilient Systems (SERENE). ACM, London, pp. 42-51 (2010)

Rodríguez, R.J., Júlvez, J., Merseguer, J.: PeabraiN: A PIPE extension for performance estimation and resource optimisation. In: Proceedings of the 12th International Conference on Application of Concurrency to System Designs (ACSD), IEEE, pp. 142-147 (2012)

Rodríguez, R.J., Júlvez, J., Merseguer, J.: On the performance estimation and resource optimisation in process Petri nets. IEEE Trans. Syst. Man Cybern. Syst. 43(6), 1385-1398 (2013)

Selic, B.: A systematic approach to domain-specific language design using UML. In: 10th IEEE International Symposium on Object and Component-Oriented Real-Time Distributed Computing (ISORC), IEEE Computer Society, Santorini Island, pp. 2-9 (2007)

Silva, M., Colom, J.: On the computation of structural synchronic invariants in P/T nets. In: Rozenberg, G. (ed.) Advances in Petri Nets 1988, Lecture Notes in Computer Science, vol. 340, pp. 386-417. Springer, Berlin (1988)

Smith, C.U., Lladó, C.M., Puigjaner, R.: Performance Model Interchange Format (PMIF 2): a comprehensive approach to queueing network model interoperability. Perform. Eval. 67(7), 548-568 (2010)

The MathWorks: Matlab. http://www.mathworks.com/. Version R2010a (2010)

Tricas, F.: Deadlock analysis, prevention and avoidance in sequential resource allocation systems. PhD thesis, Dpto. de Informática e Ingeniería de Sistemas, Universidad de Zaragoza (2003)

Université de Montréal: Stochastic simulation in Java library. http://www.iro.umontreal.ca/ simardr/ssj/ indexe.html (2014)

Woodside, M., Petriu, D.C., Petriu, D.B., Shen, H., Israr, T., Merseguer, J.: Performance by unified model analysis (PUMA). In: Proceedings of the 5th International Workshop on Software and Performance. ACM, New York, WOSP '05, pp. 1-12 (2005) 